03

\title{
Линейная трансформация поляризационных мод в намотанных на катушку spun-световодах. II. Резонансная трансформация
}

\author{
(С) Г.Б. Малыкин ${ }^{1}$, В.И. Позднякова ${ }^{2}$ \\ ${ }^{1}$ Федеральный исследовательский центр Институт прикладной физики РАН, \\ 603155 Нижний Новгород, Россия \\ ${ }^{2}$ Институт фризики микроструктур РАН, фрилиал Федерального исследовательского центра \\ Институт прикладной фозики РАН, \\ 603950 Нижний Новгород, Россия \\ e-mail: malykin@ufp.appl.sci-nnov.ru
}

Поступила в редакцию 05.03.2018 г.

Рассчитана резонансная линейная трансформация интегральных взаимно-ортогональных поляризационных мод для типичных параметров намотанных на катушку spun-световодов для четырех случаев. В первых трех случаях попарно совпадают пространственные периоды (длины) поляризационных биений невозмущенного линейного двулучепреломления, обусловленного намоткой spun-световода на катушку, невозмущенного линейного двулучепреломления и связанного с кручением spun-световода циркулярного двулучепреломления. Показано, что во всех трех случаях имеют место ярко выраженные резонансные явления, которые заключаются в периодическом изменении эллиптичности собственных интегральных поляризационных мод spun-световодов. При этом поляризация интегральных собственных мод меняется от линейной до правоциркулярной, затем до линейной, затем до левоциркулярной, затем опять до линейной. При этом во всех трех случаях имеются некоторые качественные различия. Показано, что при определенных значениях длины spun-световод утрачивает сильное двулучепреломление. Рассмотрен также случай, когда все три указанные длины поляризационных биений равны между собой. Показано, что в этом случае эволюция интегральных поляризационных мод заметно отличается от трех предыдущих случаев. Проведен расчет эволюции состояния поляризации излучения от длины spun-световодов с ее представлением на сфере Пуанкаре. Приведена оптико-механическая аналогия резонансной линейной трансформации ортогональных поляризационных мод в spun-световодах.

DOI: $10.21883 /$ OS.2018.10.46705.66-18

\section{1. Введение}

Длина поляризационных биений в одномодовом волоконном световоде $(\mathrm{OBC})$ - это такая длина отрезка OBC, на которой состояние поляризации излучения (СПИ) на выходе отрезка полностью совпадает с его СПИ на входе отрезка. В общем случае длина поляризационных биений зависит от длины волны света, что приводит к деполяризации немонохроматического излучения в ОВС [1] и расплыванию коротких световых импульсов в связных (телекоммуникационных) ОВС [2,3]. В ОВС могут иметь место линейное и циркулярное двулучепреломление различной природы, и каждому из них соответствует своя длина поляризационных биений.

Следует подчеркнуть, что при определенных значениях параметров намотанных на катушку spun-световодов могут не выполняться условия применимости геометрической оптики. В частности, это будет иметь место, если пространственный период намотки spun-световода на катушку (длина одного витка ОВС) много меньше длины поляризационных биений, обусловленных невозмущенным линейным двулучепреломлением. Впервые условия, при которых происходит нарушение законов геометрической оптики, были сформулированы еще в 1944 г. В.Л. Гинзбургом [4]. В [4] показано, что это явление будет иметь место, если пространственный период кручения оптической среды много меньше длины поляризационных биений, обусловленных невозмущенным линейным двулучепреломлением. Кроме того, в [4] показано, что это также будет иметь место, если на длине волны света показатели преломления оптической среды будут существенно меняться. Для наиболее общего случая эти условия сформулированы в работах [5,6]. Как показано в [6], при определенных условиях в неоднородной области среды, в частности там, где меняются поляризационные параметры ОВС, СПИ может не подчиняться приближению геометрической оптики (даже в оптической среде с медленно меняющимися свойствами), и тогда возникает явление линейного взаимодействия (линейной трансформации) поляризационных мод. Это явление и есть предмет исследования как предыдущей работы [7], так и настоящей части данной работы.

При нарушении условий применимости геометрической оптики интегральная длина поляризационных биений намотанных на катушку spun-световодов будет зависеть не только от значений невозмущенного ли- 
нейного двулучепреломления, обусловленного намоткой spun-световода на катушку, и связанного с кручением spun-световода циркулярного двулучепреломления, но и от СПИ на входе ОВС [6].

В первой части [7] данной работы была рассмотрена нерезонансная линейная трансформация интегральных ортогональных поляризационных мод в намотанных на катушку spun-световодах. В этом случае имеется три пространственных периода, т.е. три различные длины поляризационных биений: 1) $L_{b}$, обусловленная наличием невозмущенного линейного двулучепреломления ОВС вследствие внутреннего напряжения и (или) эллиптичности светонесущей жилы (кора) OBC, 2) $L_{\text {coil }}$, обусловленная линейным двулучепреломлением ОВС вследствие его изгибных механических напряжений, связанных с намоткой ОВС на катушку, 3) $L_{\mathrm{tw}}$, обусловленная циркулярным двулучепреломлением вследствие кручения ОВС при его вытяжке из заготовки. В [7] был рассмотрен ряд случаев, когда длины поляризационных биений $L_{b}, L_{\text {coil }}$ и $L_{\mathrm{tw}}$ существенно различались между собой.

Цель настоящей работы - вычисление зависимостей интегральных параметров spun-световода, а именно двулучепреломления, эллиптичности и азимута большой оси эллипса поляризации собственных поляризационных мод, от длины световода в случаях, когда две из трех величин $L_{b}, L_{\text {coil }}$ и $L_{\mathrm{tw}}$, или все три равны между собой. Будет также проведен расчет эволюции СПИ в зависимости от длины spun-световода для различных СПИ на входе световода, характеризующихся тремя ортогональными векторами Стокса. Результаты этого анализа будут представлены на сфере Пуанкаре (СП) [8-13].

Кроме того, будет показано, что существует определенная аналогия между рассматриваемой задачей и задачей о двух маятниках различной длины с резонансной механической связью, настроенной на разность собственных частот колебаний маятников, а также между рассматриваемой задачей и оптическим эффектом Коттона-Мутона.

\section{2. Метод вычисления}

Вычисления, так же как и в первой части данной работы [7], проводились методом численного моделирования матриц Джонса [9], предложенным и использованным авторами ранее в работе [1] (см. также $[8,14])$. Поскольку все необходимые для вычислений математические выражения приводятся в [7], то нет необходимости приводить их здесь повторно. Так же, как и в [7], будем пренебрегать потерями и дихроизмом в ОВС.

Расчеты проводились для четырех наборов параметров spun-световодов, которые приведены в таблице. Так же, как и в [7], был выбран внешний диаметр световода $D_{f}=125 \mu \mathrm{m}$ и источник монохроматического излучения с длиной волны света $\lambda=1.55 \mu \mathrm{m}$.

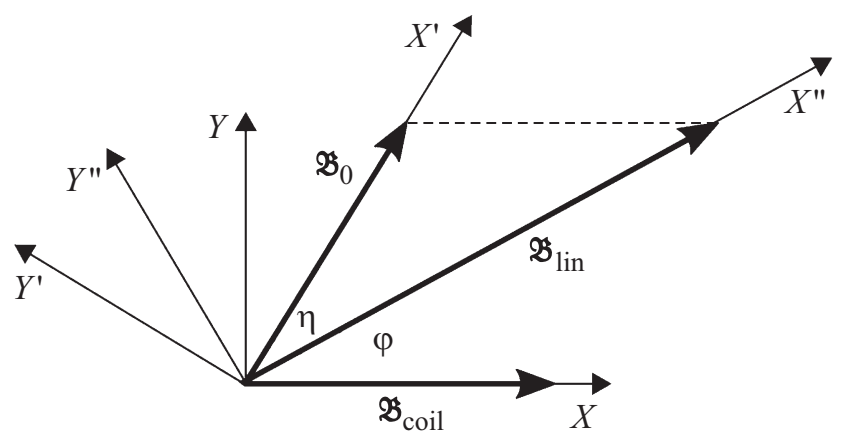

Рис. 1. Формирование линейного двулучепреломления в spunволокнах в результате векторного сложения невозмущенного линейного двулучепреломления и обусловленного намоткой на катушку линейного двулучепреломления.

Отметим, что случаи 1, 3 и 4 в таблице, когда $L_{\text {coil }}=2 \mathrm{~mm}$, реализовать практически невозможно, поскольку при этом диаметр катушки составит всего $1.64 \mathrm{~mm}$, и при намотке на такую катушку ОВС разрушится. Тем не менее для общности мы рассмотрели и эти случаи. Вычисления проводились для spunсветоводов длиной $z=40 \mathrm{~mm}$ с шагом $l=20 \mu \mathrm{m}$.

Укажем здесь на одну важную особенность резонансной линейной трансформации ортогональных поляризационных мод в намотанных на катушку spun-световодах, которая не имеет места в случае нерезонансной линейной трансформации [7]. В нерезонансном случае существенным является тот факт, что локально (на очень коротком отрезке ОВС) величины невозмущенного линейного двулучепреломления $\beta_{0}$ и обусловленного намоткой на катушку линейного двулучепреломления $\beta_{\text {coil }}$ не совпадают, т.е. $\beta_{0} \neq \beta_{\text {coil }}$. Напротив, при резонансной трансформации может иметь место такое совпадение: $\beta_{0}=\beta_{\text {coil. }}$ На рис. 1 представлено сложение векторов $\mathfrak{B}_{0}$ и $\mathfrak{B}_{\text {coil }}\left(\beta_{0 \text {, coil }}=\left|\mathfrak{B}_{0 \text {, coil }}\right|\right)$ в ситуации, когда их длины равны. Так же, как и в [7], на рис. 1 оба этих вектора лежат в плоскости $X Y$, которая ортогональна оси $Z$ OBC. Можно для удобства ввести еще две системы координат, $X^{\prime} Y^{\prime}$ и $X^{\prime \prime} Y^{\prime \prime}$, которые также лежат в плоскости $X Y$ и повернуты относительно нее соответственно на углы $\eta$ и $\varphi$. Из рис. 1 видно, что поскольку векторы $\mathfrak{B}_{0}$ и $\mathfrak{B}_{\text {coil }}$ имеют одинаковую длину, то суммарный вектор $\mathfrak{B}_{\text {lin }}=\mathfrak{B}_{0}+\mathfrak{B}_{\text {coil }}$ может иметь нулевую длину при значении угла $\eta=(2 k+1) \pi, k \in \mathbb{N}$. Иными словами, при таких углах $\eta$ линейное двулучепреломление на некотором локальном отрезке ОВС отсутствует: $\beta_{\text {lin }}=\left|\mathfrak{B}_{\text {lin }}\right|=0$. Как показано в $[4,6]$, при этом происходит нарушение законов геометрической оптики (в данном случае локальное): несмотря на наличие кручения оптической среды, вследствие отсутствия линейного двулучепреломления не будет иметь места вращение плоскости поляризации света. Возникает вопрос о применимости метода матриц Джонса для случая $\eta=(2 k+1) \pi, k \in \mathbb{N}$. 


\begin{tabular}{c|c|c|c|c|c|c|c|c|c|c}
\hline № & $\begin{array}{c}R_{\text {coil, }} \\
\mathrm{mm}\end{array}$ & $\begin{array}{c}L_{b}, \\
\mathrm{~mm}\end{array}$ & $\begin{array}{c}L_{\mathrm{tw}}, \\
\mathrm{mm}\end{array}$ & $\begin{array}{c}L_{\text {coil }}, \\
\mathrm{mm}\end{array}$ & $\begin{array}{c}\beta_{0}, \\
\mathrm{rad} / \mathrm{m}\end{array}$ & $\begin{array}{c}\beta_{\mathrm{tw}}, \\
\mathrm{rad} / \mathrm{m}\end{array}$ & $\begin{array}{c}\beta_{\text {coil }}, \\
\mathrm{rad} / \mathrm{m}\end{array}$ & $\Delta n_{0}$ & $\Delta n_{\mathrm{tw}}$ & $\Delta n_{\text {coil }}$ \\
\hline 1 & 0.82 & 2 & 10 & 2 & $3.14 \cdot 10^{3}$ & $6.28 \cdot 10^{2}$ & $3.14 \cdot 10^{3}$ & $7.75 \cdot 10^{-4}$ & $1.55 \cdot 10^{-4}$ & $7.75 \cdot 10^{-4}$ \\
2 & 1.84 & 2 & 2 & 10 & $3.14 \cdot 10^{3}$ & $3.14 \cdot 10^{3}$ & $6.28 \cdot 10^{2}$ & $7.75 \cdot 10^{-4}$ & $7.75 \cdot 10^{-4}$ & $1.55 \cdot 10^{-4}$ \\
3 & 0.82 & 10 & 2 & 2 & $6.28 \cdot 10^{2}$ & $3.14 \cdot 10^{3}$ & $3.14 \cdot 10^{3}$ & $1.55 \cdot 10^{-4}$ & $7.75 \cdot 10^{-4}$ & $7.75 \cdot 10^{-4}$ \\
4 & 0.82 & 2 & 2 & 2 & $3.14 \cdot 10^{3}$ & $3.14 \cdot 10^{3}$ & $3.14 \cdot 10^{3}$ & $7.75 \cdot 10^{-4}$ & $7.75 \cdot 10^{-4}$ & $7.75 \cdot 10^{-4}$
\end{tabular}

Если рассмотреть выражения (5)-(7) и (9) из первой части данной работы [7], то очевидно, что при отсутствии линейного двулучепреломления произведение матриц $T(\rho)$ и $M_{0}^{e}$ есть единичная матрица, поскольку при $g=0$ (что имеет место для spun-световодов) $\rho=\Theta z$, а $\delta=2 \Theta z$. Единичная матрица Джонса описывает изотропную среду, в которой нет вращения плоскости линейной поляризации света. Это соответствует результатам [4-6], где показано, что для вращения плоскости линейной поляризации света недостаточно механического кручения среды, нужно еще наличие некоторого линейного двулучепреломления. Следовательно, метод матриц Джонса позволяет проводить вычисления даже и в этом случае.

Bсе рассмотренное выше относится к локальному двулучепреломлению, т. е. двулучепреломлению на очень коротком отрезке намотанного на катушку spunсветовода. Метод вычисления интегральных характеристик таких световодов, двулучепреломления, эллиптичности и азимута большой оси эллипса собственных поляризационных мод подробно рассмотрен в работе [7].

\section{3. Результаты расчета эволюции интегральных параметров поляризационных мод и интегрального двулучепреломления намотанных на катушку одномодовых spun-световодов}

На рис. 2-5 представлены результаты численного моделирования зависимостей интегральных характеристик spun-световодов, эллиптического двулучепреломления $\beta_{\Sigma}^{e}(z)$, эллиптичности собственных поляризационных мод $\chi_{\Sigma}(z)$ и азимута большой оси эллипса $\alpha_{\Sigma}(z)$ от длины волокна $z$ для всех четырех случаев, представленных в таблице. Так же, как и в [7], зависимости $\beta_{\Sigma}^{e}(z)$ и $\chi_{\Sigma}(z)$ приводятся в системе координат, сопровождающей кручение световода, а $\alpha_{\Sigma}(z)$ - в невращающейся системе координат.

На рис. $2-5, a, b$ изображены зависимости интегрального эллиптического двулучепреломления $\beta_{\Sigma}^{e}$ от длины spun-световода $z$ в линейном и логарифмическом масштабах по оси ординат. Рис. $2-5, b$ иллюстрируют поведение зависимостей $\beta_{\Sigma}^{e}(z)$ для тех участков длины
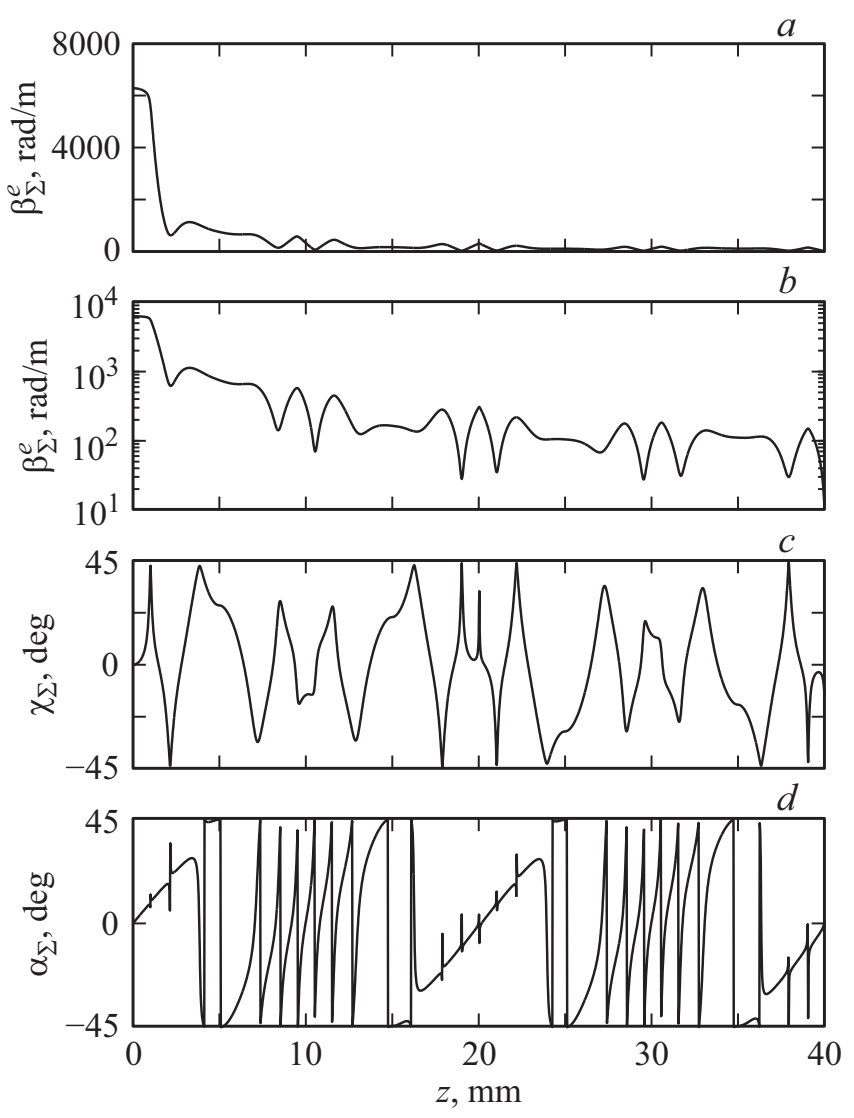

Рис. 2. Интегральные зависимости эллиптического двулучепреломления $\beta_{\Sigma}^{e}$ в линейном $(a)$ и логарифмическом $(b)$ масштабах, эллиптичности $\chi_{\Sigma}(c)$ и азимута большой оси эллипса поляризации $\alpha_{\Sigma}(d)$ от длины spun-световода $z$ с периодами собственных биений $L_{b}=2 \mathrm{~mm}$, кручения $L_{\mathrm{tw}}=10 \mathrm{~mm}$ и намотки на катушку $L_{\text {coil }}=2 \mathrm{~mm}$.

$\mathrm{OBC} z$, для которых величина $\beta_{\Sigma}^{e}$ очень мала по сравнению с величиной $\beta_{\Sigma}^{e}(0)$. Из рис. $2-5, a$ видно, что при $z=0$ величина $\beta_{\Sigma}^{e}$ принимает некоторое довольно большое значение, по порядку величины сопоставимое с $\beta_{0}$. Следует отметить, что, несмотря на то, что, как следует из таблицы, величины $\beta_{0}$ для рис. 2,3 и 5 составляют $3.14 \cdot 10^{3} \mathrm{rad} / \mathrm{m}$, а для рис. 4 всего $6.28 \cdot 10^{2} \mathrm{rad} / \mathrm{m}$, величины $\beta_{\Sigma}^{e}(0)$ составляют $\sim 6 \cdot 10^{3} \mathrm{rad} / \mathrm{m}$ для рис. 2,5 и $\sim 4 \cdot 10^{3} \mathrm{rad} / \mathrm{m}$ для рис. 3,4 . Для объяснения этого факта требуется получить хотя бы приближенное решение соответствующего дифференциального уравнения Риккати, которое, как отмечено в [7], в общем случае не имеет решения в квадратурах. Можно, однако, ка- 

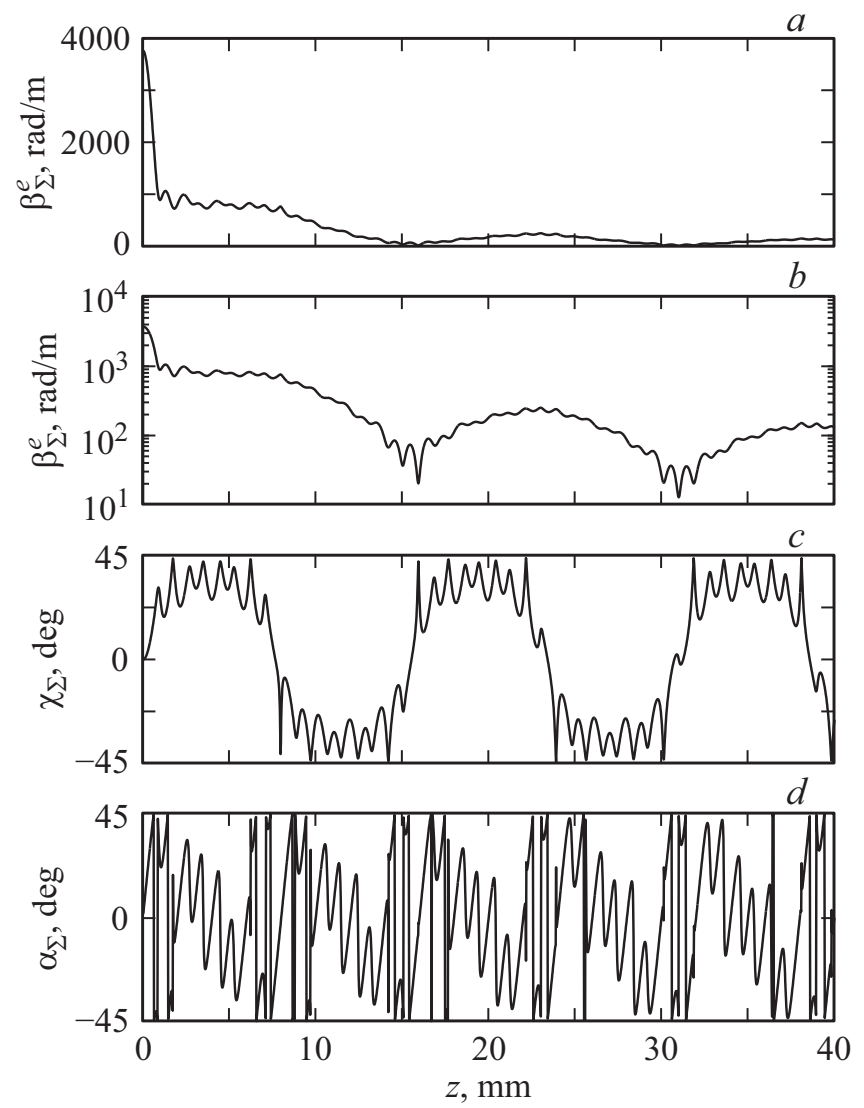

Рис. 3. Интегральные зависимости эллиптического двулучепреломления $\beta_{\Sigma}^{e}$ в линейном $(a)$ и логарифмическом $(b)$ масштабах, эллиптичности $\chi_{\Sigma}(c)$ и азимута большой оси эллипса поляризации $\alpha_{\Sigma}(d)$ от длины spun-световода $z$ с периодами собственных биений $L_{b}=2 \mathrm{~mm}$, кручения $L_{\mathrm{tw}}=2 \mathrm{~mm}$ и намотки на катушку $L_{\text {coil }}=10 \mathrm{~mm}$.

чественно пояснить поведение зависимости $\beta_{\Sigma}^{e}(z)$. Из рис. 1 видно, что величина локального линейного двулучепреломления $\beta_{\text {lin }}(z)$ равна длине суммы векторов $\mathfrak{B}_{0}(z)$ и $\mathfrak{B}_{\text {coil }}$ (см. выражение $(10)$ из работы [7]: $\left.\beta_{\text {lin }}(z)=\sqrt{\beta_{0}^{2}+\beta_{\text {coil }}^{2}+2 \beta_{0} \beta_{\text {coil }} \cos \eta(z)}\right)$. Поскольку, как следует из рис. 1 , вектор $\mathfrak{B}_{\text {coil }}$ неподвижен, а вектор $\mathfrak{B}_{0}$ вращается с ростом $z$, и в некоторых резонансных случаях $\beta_{0}=\beta_{\text {coil }}$ (рис. 2 и 5), то очевидно, что периодически с увеличением $z$ величина $\beta_{\operatorname{lin}}(z)$ обращается в ноль. Однако зависимость интегрального двулучепреломления $\beta_{\Sigma}^{e}(z)$ связана с зависимостью локального линейного двулучепреломления $\beta_{\text {lin }}(z)$ довольно сложным образом (см. (10)-(14) и (16)-(18) работы [7]), где фигурирует также величина $\beta_{\mathrm{tw}}$. Вследствие этого на рис. 2 и 5 величина $\beta_{\Sigma}^{e}(z)$ при увеличении $z$ не достигает нуля, а доходит до сравнительно небольших значений порядка $10 \mathrm{rad} / \mathrm{m}$. Величина $\beta_{\Sigma}^{e}(z)$ с ростом $z$ сразу же резко снижается, а затем с небольшими квазигармоническими осцилляциями выходит на некоторый предельный уровень. Здесь следует отметить одну важную особенность резонансной трансформации поляризационных
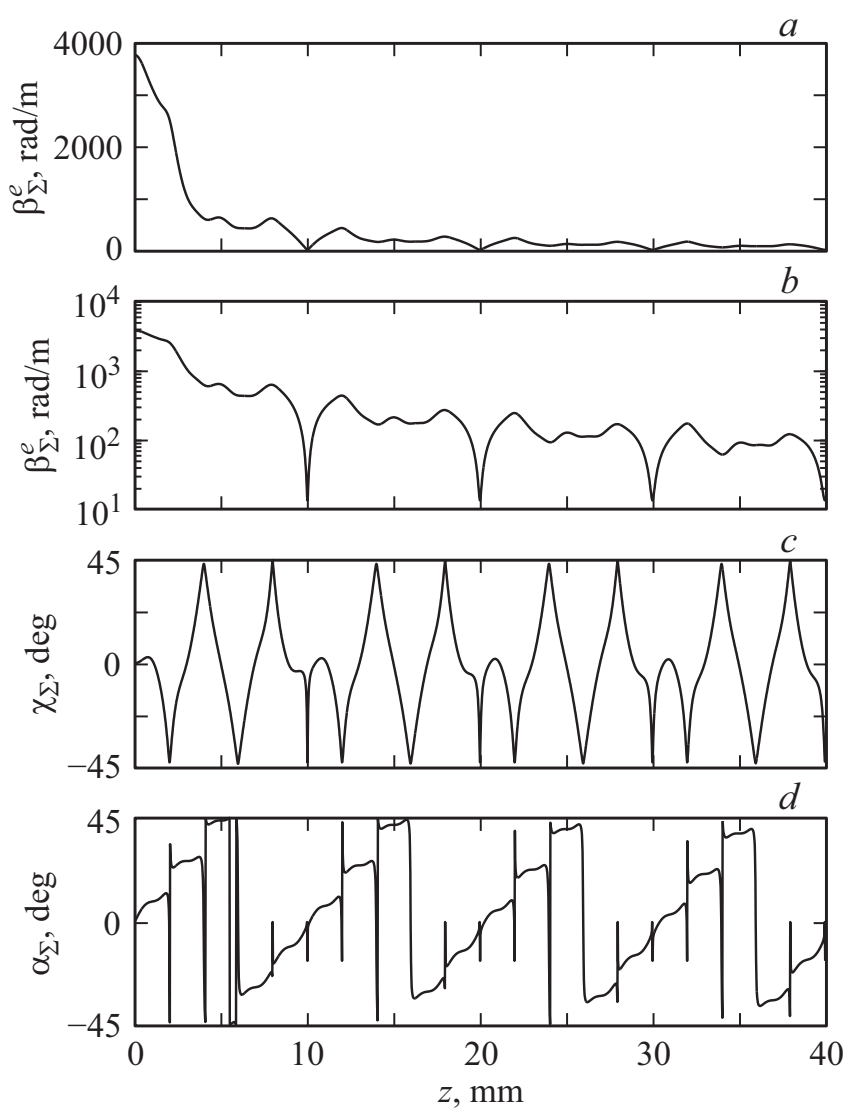

Рис. 4. Интегральные зависимости эллиптического двулучепреломления $\beta_{\Sigma}^{e}$ в линейном $(a)$ и логарифмическом $(b)$ масштабах, эллиптичности $\chi_{\Sigma}(c)$ и азимута большой оси эллипса поляризации $\alpha_{\Sigma}(d)$ от длины spun-световода $z$ с периодами собственных биений $L_{b}=10 \mathrm{~mm}$, кручения $L_{\mathrm{tw}}=2 \mathrm{~mm}$ и намотки на катушку $L_{\text {coil }}=2 \mathrm{~mm}$.

мод: в отличие от нерезонансного случая [7], здесь имеет место не один, а два периода квазигармонических осцилляций. Из этого, в частности, следует, что кручение spun-световодов как в нерезонансных [7], так и в резонансных случаях подавляет их интегральное эллиптическое двулучепреломление $\beta_{\Sigma}^{e}$. Однако в резонансных случаях это подавление значительно сильнее.

На рис. 2-5, с представлены зависимости интегральной эллиптичности $\chi_{\Sigma}$ одной из собственных поляризационных мод ${ }^{1}$ spun-световодов от длины spunсветовода $z$. Видно, что при $z=0$ для всех четыpex случаев так же, как и в нерезонансном случае [7], $\chi_{\Sigma}(0)=0$, т.е. собственные поляризационные моды spun-световодов при $z=0$ являются линейными. С ростом $z$ зависимость $\chi_{\Sigma}(z)$ ведет себя совершенно не так, как в нерезонансном случае [7], причем для всех

\footnotetext{
${ }^{1}$ Вторая поляризационная мода характеризуется эллипсом с противоположным первой интегральной поляризационной моде направлением обхода, причем главные оси эллипсов ортогональны. При этом зависимости $\chi_{\Sigma}(z)$ для первой и второй собственных мод являются инвертированными относительно оси абсцисс.
} 

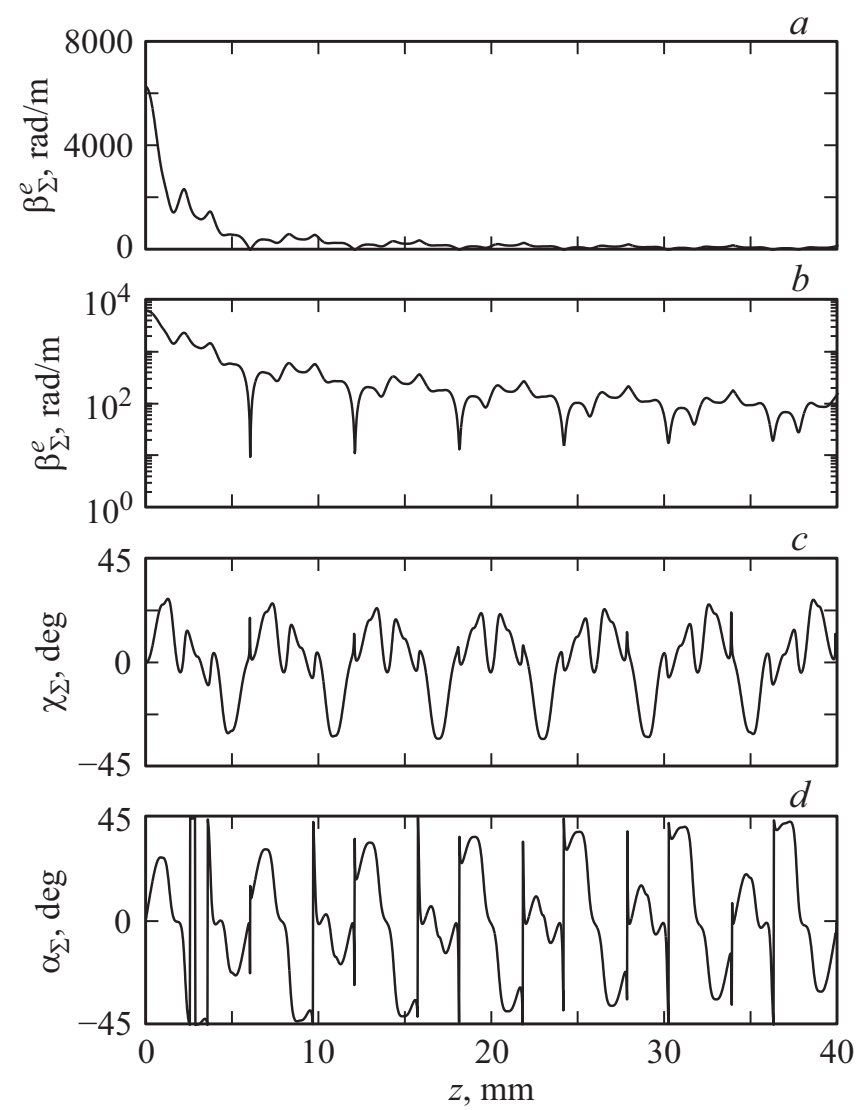

Рис. 5. Интегральные зависимости эллиптического двулучепреломления $\beta_{\Sigma}^{e}$ в линейном $(a)$ и логарифмическом $(b)$ масштабах, эллиптичности $\chi_{\Sigma}(c)$ и азимута большой оси эллипса поляризации $\alpha_{\Sigma}(d)$ от длины spun-световода $z$ с периодами собственных биений $L_{b}=2 \mathrm{~mm}$, кручения $L_{\mathrm{tw}}=2 \mathrm{~mm}$ и намотки на катушку $L_{\text {coil }}=2 \mathrm{~mm}$.

четырех случаев по-разному. Из рис. 2, с и 4, $c$ видно, что зависимость $\chi_{\Sigma}(z)$ имеет квазипериодический характер, однако и период, и амплитуда этой зависимости меняются довольно хаотическим образом. На рис. 3, $c$ и 5, зависимость $\chi_{\Sigma}(z)$ несколько ближе к гармонической, чем на рис. 2 и 4, но на эту почти гармоническую зависимость накладывается еще одна близкая к гармонической зависимость с существенно меньшими амплитудой и пространственным периодом. Основное отличие поведения зависимостей $\chi_{\Sigma}(z)$ для нерезонансного случая [7] и для рассматриваемых в данной работе резонансных случаев заключается в следующем. Как следует из результатов [7], при $z \rightarrow \infty \chi_{\Sigma}(z) \rightarrow \pi / 4$, причем это стремление носит асимптотический характер, хотя и с некоторыми незначительными осцилляциями. Таким образом, в нерезонансном случае начиная с некоторого значения $z$ собственные поляризационные моды намотанных на катушку spun-световодов становятся практически циркулярными, $\chi_{\Sigma}(z) \simeq \pi / 4[9,10]$. Из результатов данной работы следует, что для трех случаев попарного совпадения значений $L_{b}, L_{\text {coil }}$ и $L_{\mathrm{tw}}$ (обычные резонансы, рис. 2-4,c) никакой асимптотики не наблюдается.
В этом случае при изменении $z$ интегральная эллиптичность $-\pi / 4 \lesssim \chi_{\Sigma}(z) \lesssim \pi / 4$, при этом собственные поляризационные моды меняются от правоциркулярных до левоциркулярных, становясь в какой-то момент линейно поляризованными. В случае тройного резонанса (рис. 5,c) отличие от обычных резонансов заключается только в том, что эллиптичность интегральных собственных поляризационных мод периодически меняет знак в интервале значений приблизительно $\pm \pi / 6$ или, что то же самое, отношение осей эллипса поляризации $\left|\tan \chi_{\Sigma}(z)\right| \lesssim 1 / \sqrt{3}$

Что касается зависимостей $\alpha_{\Sigma}(z)$, представленных на рис. $2-5, d$, то для всех четырех рассматриваемых случаев они носят довольно хаотический характер и в отличие от соответствующих зависимостей из [7] не могут быть рационально описаны.

На рис. 6-9 представлены кривые эволюции СПИ на выходе намотанного на катушку spun-световода в зависимости от длины ОВС $z$ для различных СПИ на входе ${ }^{2}$, рассчитанные в соответствии с формулами (14), (15) первой части данной работы [7] в невращающйся системе координат и отображенные на СП. Расчет проводился для всех четырех spun-световодов, параметры которых приведены в таблице. Результаты показали, что кривые на СП существенно отличаются от соответствующих кривых, полученных в [7]. На рис. 6-8 большая часть кривых напоминает разнообразные циклоиды. Исключение составляют случай $S_{2}=1$ на рис. 7, где кривая носит довольно сложный характер, и случай $S_{3}=1$ на рис. 8, где кривая представляет собой большое число близких витков, охватывающих большой диаметр СП, и чисто внешне напоминает странный аттрактор [15]. Подчеркнем, что речь здесь идет о формальном сходстве, поскольку странный аттрактор может иметь место только в нелинейных системах [15], а в данной работе рассматриваются чисто линейные явления в ОВС. В случае тройного резонанса (рис. 9) кривые эволюции СПИ на СП представляют собой различные петлеобразные фигуры.

Очевидно, что практическое применение намотанных на катушку spun-световодов для случаев как обычных резонансов, так и тройного резонанса, нецелесообразно, поскольку, как следует из рис. 2-5, $a, b$, при этом нет асимптотического стремления собственных интегральных поляризационных мод к какому-то определенному значению, а СПИ на выходе таких ОВС, как следует из рис. 6-9, меняется в зависимости от их длины $z$ довольно сложным образом. Кроме того, для некоторых значений $z$ намотанный на катушку spun-световод становится ОВС с очень слабым эллиптическим двулучепреломлением (рис. $2-5, b)$ и не способен поддерживать заданное СПИ.

\footnotetext{
2 Расчет проводился для трех ортогональных СПИ входного излучения, характеризующегося ортогональными векторами Стокса $S_{1}=1, S_{2}=1$ и $S_{3}=1$, представление которых через компоненты вектора Джонса приводится в [7].
} 

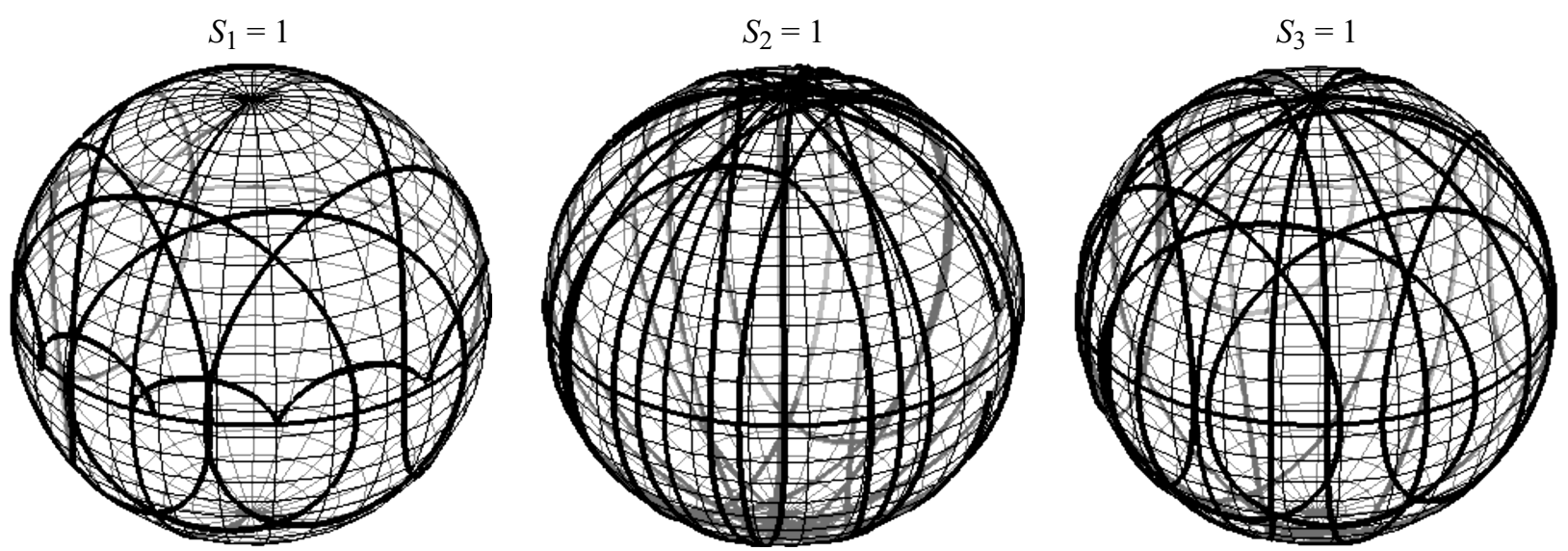

Рис. 6. Отображение на сфере Пуанкаре (СП) эволюции состояния поляризации излучения в намотанном на катушку spunсветоводе с периодами собственных биений $L_{b}=2 \mathrm{~mm}$, кручения $L_{\mathrm{tw}}=10 \mathrm{~mm}$ и намотки на катушку $L_{\text {coil }}=2 \mathrm{~mm}$ для различных поляризаций излучения на входе волокна $\left(S_{1}=1, S_{2}=1, S_{3}=1\right)$.
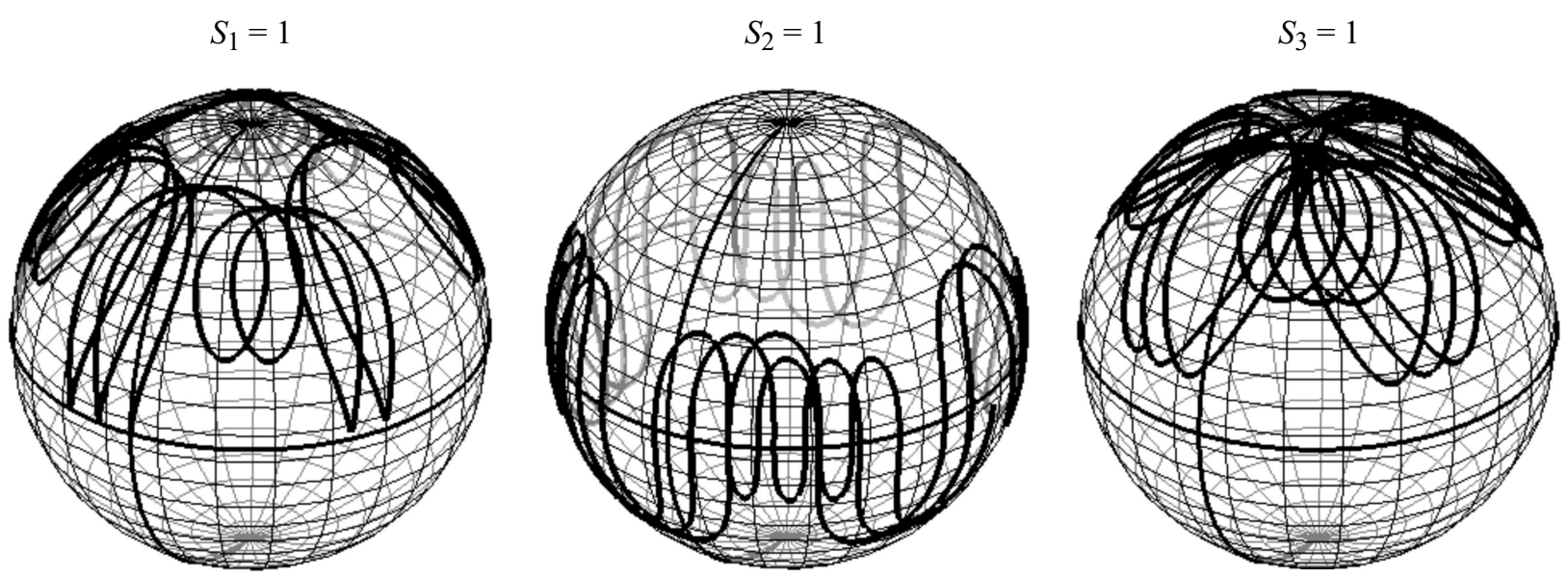

Рис. 7. Отображение на сфере Пуанкаре эволюции состояния поляризации излучения в намотанном на катушку spun-световоде с периодами собственных биений $L_{b}=2 \mathrm{~mm}$, кручения $L_{\mathrm{tw}}=2 \mathrm{~mm}$ и намотки на катушку $L_{\text {coil }}=10$ тm для различных поляризаций излучения на входе волокна $\left(S_{1}=1, S_{2}=1, S_{3}=1\right)$.

\section{4. Оптико-механическая аналогия резонансной линейной трансформации ортогональных поляризационных мод в spun-световодах}

Как было отмечено в разд. 3, для случая обычных резонансов параметров намотанных на катушку spunсветоводов одна из собственных интегральных поляризационных мод в зависимости от длины $z$ становится то правоциркулярной, то левоциркулярной (рис. 2-4, c), a ее эллиптичность пробегает все возможные значения, в том числе становится равной нулю. Соответственно, когда первая интегральная поляризационная мода имеет правую круговую поляризацию, то вторая - левую круговую поляризацию, когда первая интегральная поляризационная мода имеет горизонтальную линейную поляризацию, то вторая - вертикальную линейную поляризацию. Таким образом, по мере возрастания длины $z$ намотанных на катушку spun-световодов происходит инверсия СПИ собственных взаимно-ортогональных интегральных поляризационных мод.

В теории колебаний известно внешне довольно сходное явление: обмен энергией (амплитудой колебаний) двух маятников различной длины с резонансной механической связью, настроенной на разность собственных частот колебаний маятников ${ }^{3}$. Аналогия эта носит чисто формальный характер, поскольку обмен энергией двух маятников происходит во времени, а инверсия СПИ ортогональных интегральных поляризационных мод в пространстве, т.е. в зависимости от длины spunсветовода $z$. Это очень большое различие. Так, если периодически модулировать некоторый параметр оптической системы, например, показатель преломления, то

\footnotetext{
3 Это явление было рассмотрено более века назад Л.И. Мандельштамом в работе [16] и позднее более подробно в лекциях, прочитанных им на физфаке МГУ [17]. Также это явление освещается в $[15,18]$.
} 

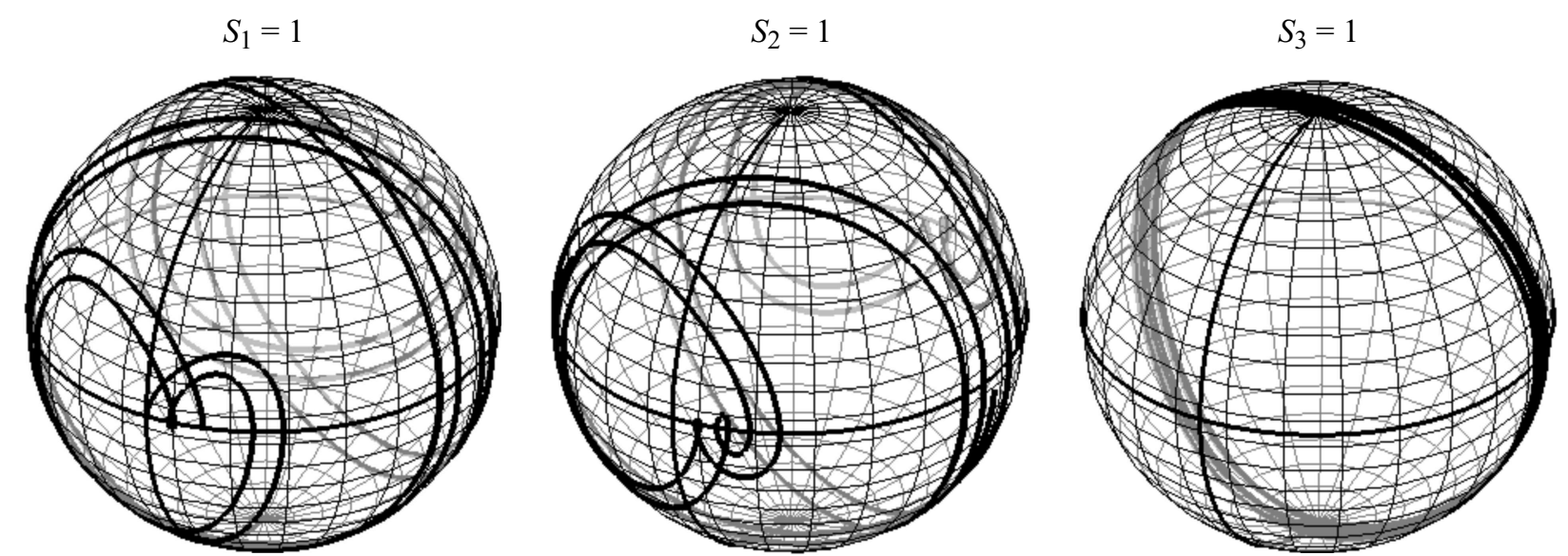

Рис. 8. Отображение на сфере Пуанкаре эволюции состояния поляризации излучения в намотанном на катушку spun-световоде с периодами собственных биений $L_{b}=10 \mathrm{~mm}$, кручения $L_{\mathrm{tw}}=2 \mathrm{~mm}$ и намотки на катушку $L_{\text {coil }}=2$ mm для различных поляризаций излучения на входе волокна $\left(S_{1}=1, S_{2}=1, S_{3}=1\right)$.
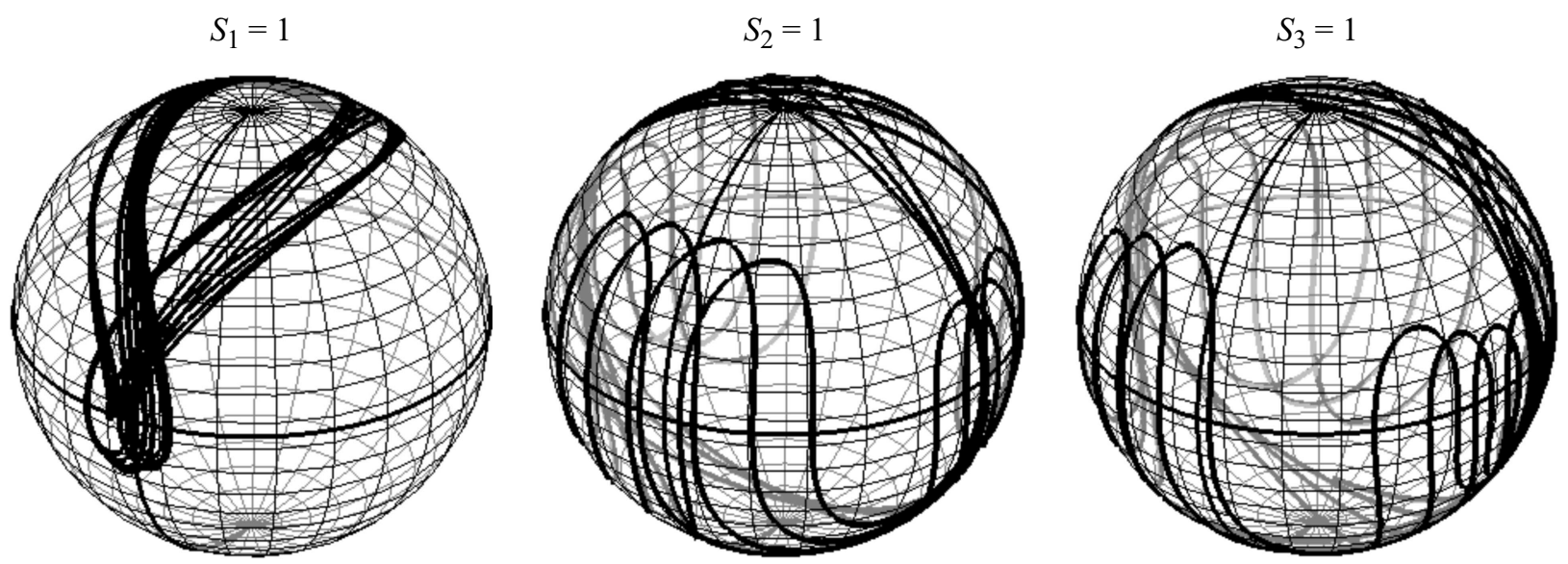

Рис. 9. Отображение на сфере Пуанкаре эволюции состояния поляризации излучения в намотанном на катушку spun-световоде с периодами собственных биений $L_{b}=2 \mathrm{~mm}$, кручения $L_{\mathrm{tw}}=2 \mathrm{~mm}$ и намотки на катушку $L_{\text {coil }}=2 \mathrm{~mm}$ для различных поляризаций излучения на входе волокна $\left(S_{1}=1, S_{2}=1, S_{3}=1\right)$.

может возникнуть параметрическое усиление света или параметрическая генерация когерентного оптического излучения. Если показатель преломления оптической системы промодулирован в пространстве, то никакого усиления света не возникнет, поскольку в этом случае отсутствует поступление внешней энергии в систему.

Оптическим аналогом разности собственных частот колебаний двух механических маятников является различие волновых чисел ортогональных интегральных поляризационных мод, разность которых связана с двулучепреломлением $\beta_{\Sigma}^{e}$. Остается только рассмотреть, что является аналогом механической связи двух маятников. Как показано в [1], в обычных (нескрученных) ОВС связь ортогональных поляризационных мод осуществляется на случайных неоднородностях ОВС. В [1] показано, что этими случайными неоднородностями, главным образом, являются случайные кручения осей линейного двулучепреломления ОВС, возникшие в процессе его вытяжки из заготовки. В данной работе, так же, как и в [7], мы не рассматриваем эти случайные кручения, поскольку их влияние практически полностью подавляется сильным постоянным кручением осей линейного двулучепреломления spun-световодов [7,8,14,19]. Тем не менее, как следует из рис. 1 (см. также (11) и (12) в [7]), и в рассматриваемом случае имеет место неравномерное вращение локальных осей линейного двулучепреломления spun-световодов: азимут $\varphi(z)$ вектора суммарного локального двулучепреломления $\mathfrak{B}_{\operatorname{lin}}$ вращается неравномерно при изменении $z$. Это и приводит к связи ортогональных интегральных поляризационных мод.

Как было отмечено в первой части данной работы [7], рассматриваемое явление инверсии СПИ собственных взаимно-ортогональных интегральных поляризационных мод имеет еще один аналог, оптический. Это эффект Коттона-Мутона, который возникает под действием поперечного по отношению к направлению светового луча магнитного поля и также приводит к осцилляциям зависимости отношения $E_{y} / E_{x}$ от длины оптической среды. 
Циркулярное двулучепреломление, связанное с кручением spun-световода, также имеет аналог - эффект Фарадея, но в отличие от последнего, кручение не приводит к невзаимному циркулярному двулучепреломлению.

Отметим, что и в нерезонансном случае (рис. 2 и $3, b[7])$ заметна незначительная инверсия СПИ обеих ортогональных интегральных поляризационных мод в намотанных на катушку spun-световодах, которая проявляется в периодических осцилляциях эллиптичности $\chi_{\Sigma}(z)$. Можно предположить, что и в задаче о двух маятниках при наличии слабой нерезонансной связи будет наблюдаться незначительный обмен энергией, который в отличие от рассмотренного в [15-18] случая не приведет к полному периодическому затуханию колебаний то одного, то другого маятника, а вызовет лишь некоторое периодическое изменение амплитуд их колебаний, происходящих в противофазе.

\section{5. Заключение}

Сформулируем основные результаты работы.

1. Показано, что в случае резонансной линейной трансформации собственные интегральные поляризационные моды намотанных на катушку spun-световодов при увеличении длины ОВС $z$ не стремятся асимптотически к какому-либо определенному значению. В случае обычных резонансов они становятся то правоциркулярными, то левоциркулярными, а эллиптичность собственных поляризационных мод пробегает все возможные значения, в том числе становится равной нулю. В случае тройного резонанса имеется некоторое отличие: интегральные поляризационные моды достигают некоторой эллиптичности, но никогда не становятся циркулярными.

2. Показано, что по мере увеличения длины $\mathrm{OBC} z$ происходит инверсия СПИ двух ортогональных интегральных поляризационных мод в намотанных на катушку spun-световодах.

3. Показано, что метод матриц Джонса позволяет проводить вычисления даже при нарушении условий выполнения законов геометрической оптики.

4. Показано, что имеет место формальная оптикомеханическая аналогия резонансной линейной трансформации ортогональных поляризационных мод в spunсветоводах и обменом энергией двух механических маятников различной длины с резонансной механической связью, настроенной на разность собственных частот колебаний маятников.

5. Показано, что практическое применение намотанных на катушку spun-световодов (например, для создания датчиков различных физических параметров) в случаях как обычных резонансов, так и тройного резонанса, нецелесообразно, поскольку при этом нет асимптотического стремления собственных интегральных поляризационных мод к какому-то определенному значению, а СПИ на выходе таких ОВС меняется в зависимости от их длины довольно сложным образом.
Кроме того, как указано выше, для некоторых значений $z$ невозможно поддерживать заданное СПИ. Следовательно, при проектировании датчиков на таких ОВС следует избегать совпадения или даже близких значений длин $L_{b}, L_{\text {coil }}$ и $L_{\mathrm{tw}}$.

Авторы выражают благодарность Вл.В. Кочаровскому, В.И. Некоркину и М.И. Рабиновичу за обсуждение рассматриваемой задачи, В.М. Геликонову за ряд полезных замечаний, позволивших существенно улучшить изложение результатов работы. Работа поддержана проектами по Государственному заданию № 0035-2014-0018 и № 0036-2014-0002.

\section{Список литературы}

[1] Малыкин Г.Б., Позднякова В.И., Шерешевский И.А. // Опт. и спектр. 1997. Т. 83. № 5. С. 843.

[2] Малыкин Г.Б., Позднякова В.И., // Изв. вузов. Радиофизика. 2011. Т. 54. № 4. С. 302.

[3] Малыкин Г.Б., Позднякова В.И., Шабанов Д.В. // Изв. вузов. Радиофизика. 2012. Т. 55. № 12. С. 792.

[4] Гинзбург В.Л. // ЖТФ. 1944. Т. 14. № 3. С. 181.

[5] Кравцов Ю.А., Орлов Ю.И. Геометрическая оптика неоднородных сред. М.: Наука, 1980. 304 с.

[6] Железняков В.В., Кочаровский В.В., Кочаровский Вл.В. // УФН. 1983. Т. 141. № 2. С. 257.

[7] Малыкин Г.Б., Позднякова В.И. // Опт. и спектр. 2018. T. 124. № 3. C. 359.

[8] Malykin G.B., Pozdnyakova V.I. Ring interferometry. Berlin: De Gruyter, 2013. 320 p.

[9] Shurkliff W.A. Polarized light. Cambridge, Massachusets: Harvard Univ. Press, 1962. 207 р. Перевод: Шерклифф У. Поляризованный свет. М.: Мир, 1965. 264 с.

[10] Малыкин Г.Б. // Изв. вузов. Радиофизика. 1997. Т. 40. № 3. C. 265.

[11] Малыкин Г.Б., Позднякова В.И. // УФН. 2004. Т. 174. № 3. C. 303.

[12] Малыкин Г.Б., Позднякова В.И. // Опт. и спектр. 2017. T. 122. № 1. C. 153.

[13] Малыкин Г.Б. // УФН. 2016. Т. 186. № 12. С. 1355.

[14] Малыкин Г.Б., Позднякова В.И. Поляризационные эффекты в кольцевых интерферометрах. Нижний Новгород: ИПФ РАН, 2008. 208 с.

[15] Рабинович М.И, Трубецков Д.И. Введение в теорию колебаний и волн. М.: Наука, 1984. С. 32; 374.

[16] Mandelstam L.I. Jahrbuch der drahtlosen Telegraphie und Telephonie. 1910-1911. V. 4. Р. 515. Перевод: Мандельштам Л.И. Полное собрание трудов. Т. 1. М.: Изд-во АН CCCР, 1948. С. 205.

[17] Мандельштам Л.И. Лекции по колебаниям, прочитанные в МГУ. Лекции № 23 (28.1.1931), № 24 (25.2.1931) и № 25 (7.3.1931). Мандельштам Л.И. Полное собрание трудов. Т. 4. М.: Изд-во АН СССР, 1955. С. 234.

[18] Трубецков Д.И., Рожнёв А.Г. Линейные колебания и волны. М.: Физматлит, 2001. С. 157.

[19] Малыкин Г.Б., Позднякова В.И., Шерешевский И.А. // Опт. и спектр. 2000. Т. 88. № 3. С. 477. 